\title{
Confiabilidade do Late-Life Function and Disability Instrument (LLFDI) versão português do Brasil em amostra de idosos com alta escolaridade
}

\author{
Adnaldo Paulo Cardoso ${ }^{a}$, Marisa Cotta Mancini ${ }^{\mathrm{a}, \mathrm{b}}$, Flora Pereira Guerra ${ }^{\mathrm{a}}$, \\ Leani Souza Maximo Pereirac, Marcella Guimaráes Assis ${ }^{\mathrm{a}, \mathrm{b}}$ \\ aPrograma de Pós-Graduação em Ciências da Reabilitação, Universidade Federal de Minas Gerais - UFMG, \\ Belo Horizonte, MG, Brasil. \\ ${ }^{\text {b} D e p a r t a m e n t o ~ d e ~ T e r a p i a ~ O c u p a c i o n a l, ~ U n i v e r s i d a d e ~ F e d e r a l ~ d e ~ M i n a s ~ G e r a i s ~-~ U F M G, ~}$ \\ Belo Horizonte, MG, Brasil.

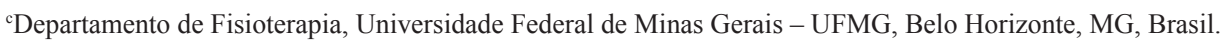

Resumo: Contextualização: O Late-Life Function and Disability Instrument (LLFDI) traduzido para o português do Brasil apresenta uma estrutura inovadora que incorpora componentes de funcionalidade e de incapacidade para avaliar idosos da comunidade. Considerando que a qualidade de um instrumento é determinada pelas suas propriedades de medida, entre elas, a confiabilidade, é aconselhável a investigação dessa propriedade após o processo de tradução e adaptação cultural. Objetivos: Avaliar a confiabilidade intra e interexaminadores da versão em português do LLFDI. Métodos: Índices de correlação intraclasse (CCI) e de concordância (CCC) foram utilizados para testar as confiabilidades intra e interexaminadores ao administrar-se o instrumento em uma amostra de 45 voluntários (idade média de 70,13 $\pm 6,88$ anos) residentes no município de Belo Horizonte, Minas Gerais. Resultados: Observaram-se altos índices de confiabilidade intraexaminador $(\mathrm{CCI}=0,91$ e CCI $=0,97)$ e interexaminadores $(\mathrm{CCC}=0,87$ e $\mathrm{CCC}=0,92$ ), respectivamente nos componentes Incapacidade (limitação total) e Função (função total) do instrumento. Conclusão: A versão traduzida para o português do Brasil do LLFDI apresentou estabilidade em ambos componentes do instrumento, mostrando-se adequada para uso no Brasil.

Palavras-chave: Idosos, Atividades Cotidianas, Funcionalidade, Incapacidade, Confiabilidade.

\section{Reliability of the Late Life Function and Disability Instrument (LLFDI) Brazilian Portuguese version in a sample of senior citizens with high educational level}

\begin{abstract}
Background: Late-Life Function and Disability Instrument (LLFDI), translated into Brazilian Portuguese, presents an innovative framework that incorporates components of functionality and disability to evaluate the elderly community. Whereas the quality of an instrument is determined by its measurement properties, including reliability, it is advisable to research such property after the instrument's process of translation and cultural adaptation. Objectives: To evaluate the intra- and inter-examiner reliability of the LLFDI Brazilian Portuguese version. Methods: Indexes of intra-class correlation (ICC) and conformity (CCC) were used to test the intra- and inter-examiner reliability by administering the instrument to a sample of 45 volunteers (average age $70.13 \pm 6.88$ years), living in Belo Horizonte, Minas Gerais state. Results: High levels of intra-examiner $(\mathrm{ICC}=0.91$ and $\mathrm{ICC}=0.97)$ and inter-examiner $(\mathrm{CCC}=0.87$ and $\mathrm{CCC}=0.92)$ reliability were observed, respectively, in the Disability (full limitation) and Function (full function) components of the instrument. Conclusion: The LLFDI Brazilian Portuguese translated version presented stability in both instrument components, being therefore suitable for use in Brazil.
\end{abstract}

Keywords: Elderly People, Daily Activities, Functionality, Disability, Reliability.

Autor para correspondência: Marcella Guimarães Assis, Programa de Pós-graduação em Ciências da Reabilitação, Universidade Federal de Minas Gerais, Av. Antônio Carlos, 6627, Pampulha, Belo Horizonte, MG, Brasil, e-mail: mga@ufmg.br

Recebido em Nov. 27, 2013; $1^{\text {a }}$ Revisão em Maio 21, 2014; 2ª Revisão em Ago. 25, 2014; Aceito em Set. 10, 2014. 


\section{Introdução}

Nos estudos sobre envelhecimento, um importante indicador de saúde é a capacidade funcional, que se refere à condição de o indivíduo viver de maneira autônoma e de se relacionar no ambiente (NOGUEIRA et al., 2010). A perda dessa capacidade resulta em incapacidade funcional, definida como a dificuldade ou necessidade de ajuda para realizar as atividades de vida diária (AVD) (ALVES et al., 2010), que englobam atividades básicas, instrumentais e avançadas. As atividades básicas de vida diária (ABVD) são definidas como atividades orientadas para o autocuidado e para a mobilidade funcional; as atividades instrumentais de vida diária (AIVD) relacionam-se ao gerenciamento da vida, do ambiente doméstico e da mobilidade na comunidade; enquanto as atividades avançadas de vida diária (AAVD) incluem os papéis sociais e o funcionamento independente na vida prática, no lazer e nas atividades produtivas (RIBEIRO; NERI, 2012).

$\mathrm{O}$ atual paradigma de funcionalidade proposto pela Classificação Internacional de Funcionalidade, Incapacidade e Saúde (CIF) (ORGANIZAÇÃO..., 2003) destaca a atividade e a participação do indivíduo nos diferentes contextos de referência, tanto no que se refere ao processo de avaliação quanto ao processo terapêutico e de acompanhamento da sua funcionalidade. Alguns autores evidenciam a importância da funcionalidade na preservação da autonomia do idoso e nas alteraçốes observadas no desempenho das AVD ao longo do processo de envelhecimento (ALVES et al., 2010; VERAS, 2009; ORGANIZAÇÃO..., 2003; HAYASE et al., 2004). A instrumentação funcional disponível, em sua maioria, centra-se principalmente no processo de incapacidade. Porém, o conhecimento do perfil de incapacidade não revela informaçôes sobre o processo de funcionalidade. Além do foco na incapacidade, os instrumentos funcionais desenvolvidos para a população idosa apresentam limitações, tais como restrição do espectro de atividades e baixa sensibilidade para documentar mudanças longitudinais (SAYERS et al., 2004; REUBEN, 1995).

Jette et al. (2002) desenvolveram o Late-Life Function and Disability Instrument (LLFDI), que é um instrumento abrangente e sensível desenvolvido para a documentaçáo de mudanças consequentes ao processo de envelhecimento. O LLFDI apresenta estrutura inovadora por incorporar componentes de incapacidade e funcionalidade para avaliar idosos da comunidade, tendo sido utilizado em diferentes estudos nas áreas de Ortopedia (SCHEELE et al., 2011), Reumatologia (GIGNAC et al., 2011),
Cardiologia (LAPIER; MIZNER, 2009), Oncologia (LOWE et al., 2009), Psiquiatria (KARP et al., 2009) e Saúde Pública (DUBOC et al., 2004). Esse instrumento já foi traduzido e validado em outros idiomas (ABIZANDA et al., 2011; MELZER et al., 2007; SAYERS et al., 2004). No Brasil, com autorização do autor, ele foi traduzido por dois grupos de pesquisa, sendo um da Universidade de Sáo Paulo (USP) (TOLDRÁ et al., 2012) e outro da Universidade Federal de Minas Gerias (UFMG), fazendo parte, nessa última instituição, do protocolo de pesquisa do Back Complaints in the Elders (BACE), estudo multicêntrico sobre dor lombar em idosos, em desenvolvimento na Austrália, Holanda e Brasil (SCHEELE et al., 2011).

É crescente o interesse, na clínica e na pesquisa científica, por instrumentos objetivos que documentem melhor o processo de reabilitação e as técnicas de tratamento (GODOTTI et al., 2006). Para qualificar um instrumento original, propriedades de medida tais como confiabilidade e validade devem ser avaliadas (SCHOLTES; TERWEE; POOLMAN, 2011). A confiabilidade é definida como o grau no qual um instrumento produz medidas livres de erros aleatórios, ou seja, o instrumento é capaz de produzir mensuraçóes estáveis e reprodutíveis (PILATTI; PEDROSO; GUTIERREZ, 2010; GODOTTI et al., 2006). Após o processo de tradução e adaptação cultural de um instrumento estrangeiro, recomenda-se a análise da confiabilidade da versão traduzida (BEATON et al., 2000; GUILLEMIN, 1995).

Alguns instrumentos que avaliam funcionalidade de idosos em atividades básicas e instrumentais de vida diária foram traduzidos e adaptados para o português do Brasil e tiveram suas propriedades de medida investigadas na população brasileira (LINO et al., 2008; SANTOS; VIRTUOSO JÚNIOR, 2008).

O presente estudo objetivou avaliar a confiabilidade da versão brasileira do LLFDI produzida pelo grupo da Universidade Federal de Minas Gerais (Anexo 1) numa população de idosos residentes na comunidade e participante de projetos de extensão universitária no município de Belo Horizonte, Minas Gerais, Brasil.

\section{Método}

\subsection{Participantes}

A amostra de conveniência foi constituída por idosos voluntários recrutados pelos pesquisadores em projetos de extensão de instituiçốes de ensino superior públicas e privadas no município de Belo Horizonte, Minas Gerais. O convite para participar 
do estudo aconteceu durante visitas realizadas aos referidos projetos. Os critérios de elegibilidade dos participantes foram: ter 60 anos ou mais e residir na comunidade. Os idosos que apresentaram déficits cognitivos detectados pelo Mini Exame do Estado Mental (MEEM) foram excluídos (BERTOLUCCI et al., 1994).

Este estudo está em conformidade com as normas internacionais para pesquisas com seres humanos e obteve aprovação do Comitê de Ética em Pesquisa da Universidade Federal de Minas Gerais (UFMG) (ETIC 0100.0.203.000-11). Todos os idosos participantes foram orientados quanto ao objetivo do estudo e assinaram o Termo de Consentimento Livre e Esclarecido.

\subsection{Instrumento}

\subsubsection{Late-Life Function and Disability Instrument (LLFDI)}

Esse instrumento foi desenvolvido por Jette et al. (2002) no Royal Center for Enhancement of Late-Life Function, Sargent College of Health and Rehabilitation Sciences da Universidade de Boston, nos EUA. Seu objetivo é avaliar a função e a incapacidade de pessoas idosas residentes na comunidade por meio de autorrelato; esse instrumento foi concebido para aplicação sob a forma de entrevista, podendo ser autoaplicável quando as condiçóes gerais de saúde o permitirem.

$\mathrm{O}$ instrumento apresenta dois componentes: Incapacidade e Função, que constituem escalas distintas. O primeiro, Incapacidade, documenta a frequência e a limitação do idoso para realização de 16 atividades de vida diária, compreendendo as atividades básicas, instrumentais e avançadas. Além dos escores totais (Frequência total e Limitação total), é possível obter um escore para cada domínio que compóe essa escala, a saber, Papel pessoal, Papel social, Papel instrumental e Papel de gerenciamento. O segundo componente, Função, informa sobre a dificuldade relatada no desempenho de 32 atividades envolvendo os membros superiores e inferiores e disponibiliza, além do escore total (Função total), a possibilidade de escores por domínios: Membros superiores, Membros inferiores básico e Membros inferiores avançado.

O LLFDI constitui instrumento abrangente e de boa compreensão na avaliação da incapacidade e da função de idosos residentes na comunidade. Ao avaliar a frequência e limitação na realização das atividades cotidianas, o LLFDI contempla uma gama de atividades que permite capturar o desempenho funcional da pessoa idosa nos ambientes doméstico e comunitário. Além disso, as limitaçôes para a realização dessas atividades são consideradas pelo LLFDI como aquelas relacionadas ao indivíduo (i.e., energia física ou mental, condiçóes de saúde) e ao ambiente (i.e., questóes de acessibilidade, problemas de transporte e circunstâncias sociais e econômicas).

O componente Função do LLFDI documenta a habilidade para realizar diferentes atividades específicas que podem fazer parte de um dia típico, envolvendo membros superiores e membros inferiores. As dificuldades para realizar essas atividades podem incluir: dor, fadiga, medo, fraqueza, dolorimentos ou outras condiçóes de saúde e incapacidade.

As cinco possibilidades de resposta para cada uma das perguntas tornam o instrumento mais sensível para documentar variaçôes nas atividades cotidianas realizadas por pessoas idosas nas escalas de Função e de Incapacidade quando comparado a outros disponíveis no idioma português do Brasil.

\subsection{Procedimentos}

O LLFDI foi aplicado sob a forma de entrevista, por dois avaliadores previamente treinados, que seguiram orientaçóes padronizadas propostas nas instruçôes iniciais do instrumento. A confiabilidade intra e interexaminadores foi avaliada em três momentos distintos: os dois primeiros com a administraçáo do instrumento por dois examinadores independentes (examinador 1 e examinador 2), num mesmo momento, e o terceiro com a reaplicaçáo do instrumento pelo examinador 1 , no intervalo de 8 a 10 dias da primeira administração.

\subsection{Análise estatística}

Os participantes foram caracterizados por medidas de tendência central (média), de dispersão (desvio-padrão) e porcentagem. O coeficiente de correlação intraclasse (ICC) quantificou a confiabilidade intraexaminador e o coeficiente de correlação de concordância (CCC) quantificou a confiabilidade interexaminador.

Os dados foram analisados pelo programa estatístico SPSS (Statistical Package for Social Sciences) versão 18.0, 2011.

\section{Resultados}

Informaçōes descritivas sobre o perfil sociodemográfico dos 45 idosos participantes deste estudo encontram-se na Tabela 1. 
Para os diferentes escores da versão em português do Brasil do LLFDI encontrou-se forte concordância intra e interexaminadores. A magnitude dos índices de confiabilidade (ICC e CCC) está apresentada nas Tabelas 2 e 3.

\section{Discussão}

A versão brasileira do LLFDI apresentou alta confiabilidade intraexaminador no componente Incapacidade (ICC $=0,95$ e 0,91) para Frequência

Tabela 1. Informações descritivas da amostra nas variáveis sociodemográficas.

\begin{tabular}{llr}
\hline & Variável & $\%$ \\
\hline Idade & $60-69$ & 53,3 \\
Média: & $70-79$ & 35,5 \\
$70,13 \pm 6,88$ & 80 e mais & 11,2 \\
Sexo & Masculino & 24,4 \\
& Feminino & 75,6 \\
Escolaridade & $1-4$ & 6,7 \\
(em anos) & $5-8$ & 17,8 \\
& $9-11$ & 24,4 \\
& 12 e mais & 51,1 \\
Estado civil & Casado ou vive com & 53,3 \\
& companheiro (a) & \\
& Solteiro (a) & 6,7 \\
& Separado (a) ou & 13,3 \\
& divorciado (a) & \\
Renda & Viúvo (a) & 26,7 \\
& Sim & 88,9 \\
Renda & Não & 11,1 \\
própria & Até 1 salário-mínimo & 2,4 \\
& 2 salários-mínimos & 31,0 \\
& 3 salários-mínimos & 4,8 \\
& 4 salários-mínimos & 16,7 \\
& 5 salários-mínimos & 45,2 \\
& ou mais & \\
\hline & & \\
\hline
\end{tabular}

total e Limitação total, respectivamente; e no componente Função (ICC = 0,93 a 0,97). Os índices de concordância interexaminadores foram fortes para os componentes Incapacidade (CCC $=0,86$ a 0,97 ) e Função (CCC $=0,88$ a 0,94$)$ (PORTNEY; WATKINS, 2009).

Esses resultados são semelhantes aos encontrados para a versão original (JETTE et al., 2002; HALEY et al., 2002) e hebraica (MELZER et al., 2007) do LLFDI. A similaridade dos resultados foi observada, principalmente, no componente Função, sendo encontrados índices de confiabilidade muito próximos aos da versão original ( $\mathrm{ICC}=0,91$ a 0,98 ) (HALEY et al., 2002) e hebraica (ICC $=0,77$ a 0,90) (MELZER et al., 2007).

O componente Incapacidade do LLFDI apresentou índices de confiabilidade superiores aos das versóes original (ICC $=0,68$ a 0,82) (JETTE et al., 2002) e hebraica (ICC $=0,63$ a 0,83) (MELZER et al., 2007) para a Frequência total e Limitação total, respectivamente. Embora superiores, os resultados se comportaram como nas outras versóes, com menor valor para a confiabilidade do domínio Papel instrumental. Os autores atribuíram essa diferença ao fato de ele ser composto por somente quatro itens (JETTE et al., 2002; MELZER et al., 2007).

A confiabilidade da versão brasileira, desenvolvida pelo grupo da Universidade Federal de Minas Gerais, apresentou elevada magnitude, o que contribui para qualificar esse instrumento (PORTNEY; WATKINS, 2009). Tal magnitude pode também refletir o elevado nível de escolaridade da população avaliada, pois mais da metade dos voluntários apresentaram nível superior completo (12 anos e mais de escolaridade) e cerca de um quarto concluiu o ensino médio (9-11 anos de escolaridade). Essas

Tabela 2. Valores do coeficiente de correlação intraclasse (ICC) e do coeficiente de correlação de concordância (CCC) intra e interexaminador do componente Incapacidade do instrumento LLFDI.

\begin{tabular}{|c|c|c|}
\hline LLFDI - Incapacidade & $\begin{array}{l}\text { Confiabilidade } \\
\text { Intraexaminador }\end{array}$ & $\begin{array}{l}\text { Confiabilidade } \\
\text { interexaminadores }\end{array}$ \\
\hline Frequência total & $\mathrm{ICC}=0,95$ & $\mathrm{CCC}=0,92$ \\
\hline Papel social & $\mathrm{ICC}=0,96$ & $\mathrm{CCC}=0,97$ \\
\hline $\mathrm{D} 1 \mathrm{a}+\mathrm{D} 2 \mathrm{a}+\mathrm{D} 3 \mathrm{a}+\mathrm{D} 5 \mathrm{a}+\mathrm{D} 6 \mathrm{a}+\mathrm{D} 9 \mathrm{a}+\mathrm{D} 11 \mathrm{a}+\mathrm{D} 12 \mathrm{a}+\mathrm{D} 14 \mathrm{a}$ & & \\
\hline $\begin{array}{l}\text { Papel pessoal } \\
\text { D4a + D7a + D8a + D10a +D13a + D15a + D16a }\end{array}$ & $\mathrm{ICC}=0,89$ & $\mathrm{CCC}=0,93$ \\
\hline Limitação total & $\mathrm{ICC}=0,91$ & $\mathrm{CCC}=0,87$ \\
\hline $\begin{array}{l}\text { Papel instrumental } \\
\mathrm{D} 2 \mathrm{~b}+\mathrm{D} 3 \mathrm{~b}+\mathrm{D} 4 \mathrm{~b}+\mathrm{D} 5 \mathrm{~b}+\mathrm{D} 6 \mathrm{~b}+\mathrm{D} 9 \mathrm{~b}+\mathrm{D} 10 \mathrm{~b}+\mathrm{D} 12 \mathrm{~b}+\mathrm{D} 13 \mathrm{~b}+ \\
\mathrm{D} 14 \mathrm{~b}+\mathrm{D} 15 \mathrm{~b}+\mathrm{D} 16 \mathrm{~b}\end{array}$ & $\mathrm{ICC}=0,91$ & $\mathrm{CCC}=0,86$ \\
\hline $\begin{array}{l}\text { Papel de gerenciamento } \\
\mathrm{D} 1 \mathrm{~b}+\mathrm{D} 7 \mathrm{~b}+\mathrm{D} 8 \mathrm{~b}+\mathrm{D} 11 \mathrm{~b}\end{array}$ & $\mathrm{ICC}=0,88$ & $\mathrm{CCC}=0,92$ \\
\hline
\end{tabular}

A letra maiúscula D refere-se a Disability, o número corresponde à ordem da questão no questionário e as letras (a ou b) equivalem, respectivamente, à frequência (a) ou limitação (b). 
Tabela 3. Valores do coeficiente de correlação intraclasse (ICC) e do coeficiente de correlação de concordância (CCC) intra e interexaminador do componente Função do instrumento LLFDI.

\begin{tabular}{lcc}
\hline \multicolumn{1}{c}{ LLFDI - Função } & $\begin{array}{c}\text { Confiabilidade } \\
\text { intraexaminador }\end{array}$ & $\begin{array}{c}\text { Confiabilidade } \\
\text { interexaminadores }\end{array}$ \\
\hline Função total & ICC $=0,97$ & CCC $=0,92$ \\
Membros superiores & ICC $=0,94$ & CCC $=0,94$ \\
F 1 F3 + F5 + F6 + F13 + F16 + F17 & ICC $=0,93$ & CCC $=0,94$ \\
Membros inferiores básico & & \\
F2 + F10 + F11 + F12 + F14 + F15 + & & \\
F18 + F21 + F22 + F23 + F25 + F26 + F28 + F31 & ICC $=0,97$ & CCC $=0,88$ \\
Membros inferiores avançado & & \\
F4 + F7 + F8 + F9 + F19 + F20 + F24 + F27 + F29 + F30 + F32 &
\end{tabular}

A letra maiúscula $\mathrm{F}$ refere-se a Function e o número, à ordem da questão no questionário.

características sugerem boa reserva cognitiva, que pode ter minimizado o viés de memória frequentemente observado em estudos com a população idosa, influenciando positivamente a qualidade das informações disponibilizadas pelos participantes.

Outro fator que possivelmente concorreu para os altos índices de confiabilidade encontrados neste estudo foi terem sido acatadas as sugestóes do comitê de especialistas durante o processo de adaptação do instrumento. Esse comitê propôs que, tendo em vista as dificuldades encontradas pela população avaliada no período de pré-teste do instrumento, fosse disponibilizado o conceito de limitação ao respondente, de modo a facilitar sua compreensão sobre a pergunta "Até que ponto você se sente limitado(a) em realizar determinada atividade?" Destaca-se ainda que os autores desta versão brasileira conceberam o "auxílio gráfico para respostas" (Anexo 2), de modo a facilitar a escolha das respostas para o componente Incapacidade. O examinador apontava na folha as opçóes e o examinado selecionava o escore correspondente à resposta.

\section{Conclusão}

A versáo traduzida do LLFDI para o português do Brasil, pelo grupo da Universidade Federal de Minas Gerais mostrou boa confiabilidade intra e interexaminadores, com elevados índices nos dois componentes dessa escala. Apesar de os autores da escala original admitirem a possibilidade de o instrumento ser autoadministrado, dado o baixo nível de escolaridade de grande parte da população idosa no Brasil, que chega a $30,7 \%$ de idosos com menos de um ano de instrução (INSTITUTO..., 2011), recomenda-se a aplicação no formato de entrevista por um examinador treinado. Sugere-se que o entrevistador leia as instruçôes iniciais do instrumento, utilize corretamente o "auxílio gráfico para respostas" e certifique-se de que o idoso compreendeu o enunciado e as perguntas.

Estudos subsequentes são necessários para investigar o desempenho dessa versão do LLFDI em diferentes grupos da populaçáo idosa brasileira, bem como em estudos de acompanhamento longitudinal, para testar a sensibilidade desse instrumento na documentação de mudanças ao longo do tempo.

\section{Referências}

ABIZANDA, P. et al. Validation of the Spanish version of the Short-Form Late-Life Function and Disability Instrument. Journal of the American Geriatrics Society, New York, v. 59, n. 5, p. 893-899, 2011.

ALVES, L. C. et al. Fatores associados à incapacidade funcional dos idosos no Brasil: análise multinível. Revista de Saúde Prública, São Paulo, v. 44, n. 3, p. 468-478, 2010.

BEATON, D. et al. Guidelines for the process of crosscultural adaptation of self-report measures. Spine, London, v. 25, n. 24, p. 3186-3191, 2000.

BERTOLUCCI, P. et al. O Mini-Exame do Estado Mental em uma populaçấo geral: impacto da escolaridade. Arquivos de Neuropsiquiatria, São Paulo, v. 52, n. 1, p. 1-7, 1994.

DUBOC, N. et al. Function and disability in late life: comparison of the Late-Life Function and Disability Instrument to the Short-Form-36 and the London Handicap Scale. Disability and Rehabilitation, London, v. 26, n. 6, p. 362-370, 2004 .

GIGNAC, M. A. M. et al. Measures of disability. Arthritis Care \& Research, Cary, v. 63, n. 11, p. 308-324, 2011.

GODOTTI, I. C.; VIEIRA, E. R.; MAGEE, D. J. Importance and clarification of measurement propertirs in rehabilitation. Revista Brasileira de Fisioterapia, São Carlos, v. 10, n. 2, p. 137-146, 2006.

GUILLEMIN, F. Cross-cultural adaptation and validation of health status measures. Scandinavian Journal of Rheumatology, Aarhus, v. 24, n. 2, p. 61-63, 1995. 
http://dx.doi.org/10.3109/03009749509099285. PMid:7747144

HALEY, S. M. et al. Late Life Function and Disability Instrument: II. Development and evaluation of the function component. The Journal of Gerontology: Biological Sciences and Medical Sciences, Washington, v. 57, n. 4, p. M217-M222, 2002. http://dx.doi.org/10.1093/ gerona/57.4.M217. PMid:11909886

HAYASE, D. et al. Age-related changes in activities of daily living ability. Australian Occupational Therapy Journal, Richmond, v. 51, n. 4, p. 192-198, 2004.

INSTITUTO BRASILEIRO DE GEOGRAFIA E ESTATÍSTICA - IBGE. Pesquisa Nacional de Amostra de Domicílio (PNAD). Rio de Janeiro, 2001.

JETTE, A. M. et al. Late life function and disability instrument: I. Development and evaluation of the disability component. The Journal of Gerontology: Biological Sciences and Medical Sciences, Washington, v. 57, n. 4, p. 209-216, 2002. http://dx.doi.org/10.1093/ gerona/57.4.M209. PMid:11909885

KARP, J. F. et al. Use of the late-life function and disability instrument to assess disability in major depression. Journal of the American Geriatrics Society, New York, v. 57, n. 9, p. 1612-1619, 2009. http://dx.doi.org/10.1111/ j.1532-5415.2009.02398.x. PMid:19682111

LAPIER, T. K.; MIZNER, R. Outcome measures in cardiopulmonary physical therapy: focus on the Late Life Function and Disability Instrument (LLFDI). Cardiopulmonary Physical Therapy Journal, St. Louis, v. 20, n. 2, p. 32-35, 2009.

LINO, V. T. S. et al. Adaptação transcultural da Escala de Independência em Atividades da Vida Diária (Escala de Katz). Cadernos de Saúde Pública, Rio de Janeiro, v. 24, n. 1, p. 103-112, 2008.

LOWE, S. S. et al. Associations between physical activity and quality of life in cancer patients receiving palliative care: a pilot survey. Journal of Pain and Symptom Management, New York, v. 38, n. 5, p. 785-796, 2009. http:// dx.doi.org/10.1016/j.jpainsymman.2009.03.006. PMid:19775864

MELZER, I. et al. Relationship between self-reported function and disability and balance performance measures in the elderly. Journal of Rehabilitation Research \& Development, Washington, v. 44, n. 5, p. 685-691, 2007. http://dx.doi.org/10.1682/JRRD.2006.10.0133. PMid:17943680
NOGUEIRA, S. L. et al. Fatores determinantes da capacidade funcional de idosos longevos. Revista Brasileira de Fisioterapia, São Carlos, v. 14, n. 4, p. 322-329, 2010.

ORGANIZAÇÃO MUNDIAL DE SAÚDE - OMS. Classificação Internacional de Funcionalidade, Incapacidade e Saúde. São Paulo: Edusp, 2003.

PILATTI, L. A.; PEDROSO, B.; GUTIERREZ, G. L. Propriedades psicométricas de instrumentos de avaliação. Revista Brasileira de Ensino em Ciência e Tecnologia, Ponta Grossa, v. 3, n. 1, p. 81-91, 2010.

PORTNEY, L. G.; WATKINS, M. P. Foundations of Clinical Research: applications to practice. New Jersey: Upper Saddle River, 2009.

REUBEN, D. B. What's wrong with ADLs? Journal of the American Geriatrics Society, New York, v. 43, n. 8, p. 936-937, 1995. http://dx.doi. org/10.1111/j.1532-5415.1995.tb05540.x. PMid:7636105

RIBEIRO, L. H. M.; NERI, A. L. Exercícios físicos, força muscular e atividades de vida diária em mulheres idosas. Ciência \& Saúde Coletiva, Rio de Janeiro, v. 17, n. 8, p. 2169-2180, 2012.

SANTOS, R. L.; VIRTUOSO JÚNIOR, J. S. Confiabilidade da versão brasileira da Escala de Atividades Instrumentais da Vida Diária. Revista Brasileira em Promoção da Saúde, Fortaleza, v. 21, n. 4, p. 290-296, 2008.

SAYERS, S. P. et al. Validation of the Late-Life Function and Disability Instrument. The Journal of Gerontology: Biological Sciences and Medical Sciences, Washington, v. 52, n. 9, p.1554-1559, 2004. http://dx.doi.org/10.1111/ j.1532-5415.2004.52422.x. PMid:15341561

SCHEELE, J. et al. Back Complaints in the Elders (BACE); design of cohort studies in primary care: an international consortium. BMC Musculoskeletal Disorders, London, v. 12, p. 193-201, 2011.

SCHOLTES, V. A.; TERWEE, C. B.; POOLMAN, R. W. What makes a measurement instrument valid and reliable? Injury-International Journal Care of the Injured, New York, v. 42, n. 3, p. 236-240, 2011.

TOLDRÁ, R. C. et al. Adaptação transcultural do Latelife Function and Disability Instrument para o português brasileiro. Revista de Terapia Ocupacional da Universidade de São Paulo, São Paulo, v. 23, n. 1, p. 52-61, 2012.

VERAS, R. Envelhecimento populacional contemporâneo: demandas, desafios e inovaçōes. Revista de Saúde Pública, Rio de Janeiro, v. 43, n. 3, p. 548-554, 2009. 


\section{Contribuição dos Autores}

Adnaldo Paulo Cardoso: Concepção do texto, coleta de dados, organização e análise dos resultados, redação do texto. Flora Pereira Guerra: Coleta de dados. Marisa Cotta Mancini e Leani Souza Maximo Pereira: Interpretação dos resultados, revisão. Marcella Guimarães Assis: Concepção do texto, interpretação dos resultados, redação do texto, revisão. Todos os autores aprovaram a versão final do texto. 
Anexo 1

\section{Late-Life FDI: Componente de Incapacidade ${ }^{1}$}

\section{INSTRUÇÕES PARA AS QUESTÕES SOBRE INCAPACIDADE}

Neste conjunto de questôes, eu perguntarei a você sobre coisas do dia a dia que você faz nesse momento da sua vida. Há duas partes para cada questáo.

Primeiro, eu perguntarei a você Com que frequência você faz uma determinada atividade.

Em seguida, eu lhe perguntarei Até que ponto você se sente limitado(a) em fazer esta atividade.

Explique cada questão e as opçóes de resposta subsequentes

Para a primeira questão (Com que frequência você faz a atividade?), por favor, escolha uma entre as seguintes respostas:

\section{Com muita frequência}

\section{Com frequência}

De vez em quando

\section{Quase nunca}

\section{Nunca}

[Mostre o auxílio visual para o entrevistado]

Para a segunda questão (Até que ponto você se sente limitado(a) em fazer a atividade?), por favor, escolha uma entre as seguintes respostas:

\section{De jeito nenhum}

\section{Um pouco}

\section{Mais ou menos}

\section{Muito \\ Completamente}

[Mostre o auxílio visual para o entrevistado]

\section{Limitações são dificuldades que podemos ter para realizar uma atividade.}

Por exemplo, você pode se sentir limitado (a) por causa de sua saúde, ou porque a atividade exige muita energia mental e física. Por favor, lembre-se de que você também pode se sentir limitado (a) por fatores externos a você. Seu ambiente pode restringi-lo (a) de fazer as atividades: por exemplo, questôes relacionadas a transporte, acessibilidade e circunstâncias sociais e econômicas podem limitá-lo (a) de fazer coisas que você gostaria de fazer. Pense em todos esses fatores quando responder a esta parte.

Para cada questão, por favor, selecione a resposta que mais se aproximar da forma como você vem se sentindo.

Vamos começar...

\footnotetext{
${ }^{1}$ Esta versão traduzida para o português do Brasil foi parte integrante do projeto de mestrado de Adnaldo Paulo Cardoso, orientado pela professora doutora Marcella Guimarães Assis, do Programa de Pós-Graduação em Ciências da Reabilitação, Escola de Educação Física, Fisioterapia e Terapia Ocupacional, Universidade Federal de Minas Gerais, 2013.
} 


\section{Questões sobre Incapacidade}

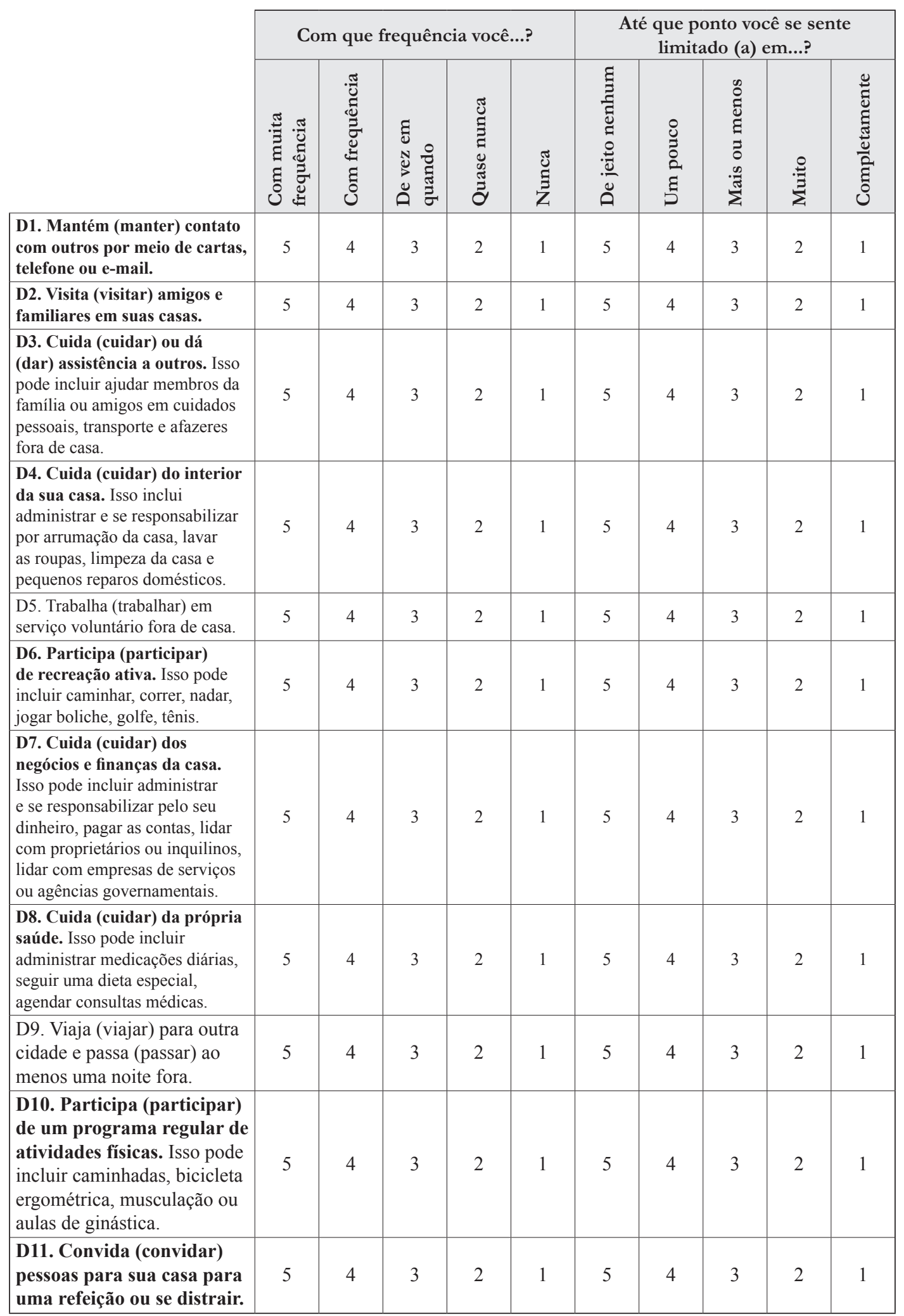


Questões sobre Incapacidade - Continuação...

\begin{tabular}{|c|c|c|c|c|c|c|c|c|c|c|}
\hline & \multicolumn{5}{|c|}{ Com que frequência você...? } & \multicolumn{5}{|c|}{$\begin{array}{c}\text { Até que ponto você se sente } \\
\text { limitado (a) em...? }\end{array}$} \\
\hline & 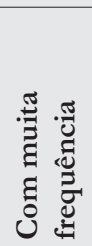 & 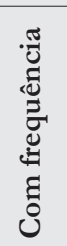 & 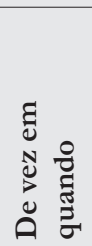 & 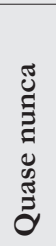 & 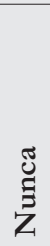 & 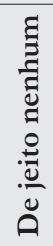 & $\begin{array}{l}8 \\
\vdots \\
\vdots \\
\vdots \\
\vdots\end{array}$ & 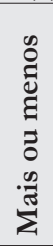 & 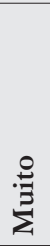 & 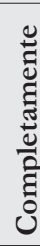 \\
\hline $\begin{array}{l}\text { D12. Sai (sair) com } \\
\text { outras pessoas para locais } \\
\text { públicos como restaurantes } \\
\text { ou cinemas. }\end{array}$ & 5 & 4 & 3 & 2 & 1 & 5 & 4 & 3 & 2 & 1 \\
\hline $\begin{array}{l}\text { D13. Cuida (cuidar) de } \\
\text { suas necessidades de } \\
\text { cuidados pessoais. Isso } \\
\text { inclui tomar banho, vestir-se } \\
\text { e higiene pessoal. }\end{array}$ & 5 & 4 & 3 & 2 & 1 & 5 & 4 & 3 & 2 & 1 \\
\hline $\begin{array}{l}\text { D14. Participa (participar) } \\
\text { de atividades sociais } \\
\text { organizadas. Isso pode } \\
\text { incluir agremiações, jogos } \\
\text { de cartas, eventos de grupos } \\
\text { de terceira idade, grupos } \\
\text { religiosos ou comunitários. }\end{array}$ & 5 & 4 & 3 & 2 & 1 & 5 & 4 & 3 & 2 & 1 \\
\hline $\begin{array}{l}\text { D15. Realiza (realizar) } \\
\text { afazeres nas proximidades } \\
\text { de sua casa. Isso pode } \\
\text { incluir se responsabilizar } \\
\text { e lidar com a compra de } \\
\text { comida, itens pessoais e } \\
\text { ir ao banco, biblioteca ou } \\
\text { lavanderia. }\end{array}$ & 5 & 4 & 3 & 2 & 1 & 5 & 4 & 3 & 2 & 1 \\
\hline $\begin{array}{l}\text { D16. Prepara (preparar) } \\
\text { as próprias refeições. Isso } \\
\text { inclui planejar, cozinhar, } \\
\text { servir e limpar. }\end{array}$ & 5 & 4 & 3 & 2 & 1 & 5 & 4 & 3 & 2 & 1 \\
\hline
\end{tabular}

\section{Late-Life FDI: Componente de Função ${ }^{1}$}

\section{INSTRUÇÕES PARA AS QUESTÕES FUNCIONAIS}

Nesta seção, eu perguntarei a você sobre sua habilidade em realizar atividades específicas como parte de sua rotina diária. Estou interessado na sua percepção de suas habilidades para realizar atividades em um dia típico. Não é importante que você realmente faça a atividade diariamente. Na verdade, eu posso mencionar algumas atividades que você não faz de jeito nenhum. Ainda assim, você pode responder às perguntas avaliando o quanto você acha que seria difícil para você realizá-las em um dia qualquer.

Fatores que influenciam o nível de dificuldade que você tem podem incluir: dor, fadiga, medo, fraqueza, dolorimentos, adoecimentos, condiçóes de saúde ou incapacidade.

Eu quero saber o quanto é difícil para você realizar a atividade sem a ajuda de outra pessoa e sem o auxílio de uma bengala, andador ou qualquer outro dispositivo de auxílio para a locomoção (como cadeiras de rodas ou carrinhos motorizados). 
Nota pessoal ao entrevistador

Para os itens de Função, o uso de um apoio fixo é aceitável (por exemplo, segurar-se em móveis, paredes), a não ser que esteja especificado de outra forma no item.

[Mostre o auxílio visual para o entrevistado]

Por favor, escolha uma entre as seguintes respostas:

\section{Nenhuma}

\section{Pouca}

\section{Alguma}

\section{Muita}

\section{Não consigo fazer}

Vamos começar...

\section{Questões sobre Função}

\begin{tabular}{|c|c|c|c|c|c|}
\hline $\begin{array}{l}\text { Quanta dificuldade você tem para...? } \\
\text { (Lembre-se que isso é sem a ajuda de outras } \\
\text { pessoas e sem o uso de qualquer dispositivo de } \\
\text { auxílio para a locomoção.) }\end{array}$ & 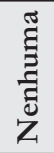 & 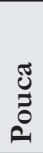 & 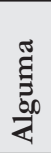 & 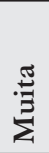 & Z \\
\hline $\begin{array}{l}\text { F1. Abrir a tampa de um pote que não foi previamente } \\
\text { aberto sem usar dispositivos de auxílio. }\end{array}$ & 5 & 4 & 3 & 2 & 1 \\
\hline $\begin{array}{l}\text { F2. Subir e descer um lance de escadas internas } \\
\text { usando o corrimão }\end{array}$ & 5 & 4 & 3 & 2 & 1 \\
\hline $\begin{array}{l}\text { F3. Colocar e tirar calças compridas (incluindo lidar } \\
\text { com fechos). }\end{array}$ & 5 & 4 & 3 & 2 & 1 \\
\hline F4. Correr 800 metros ou mais. & 5 & 4 & 3 & 2 & 1 \\
\hline $\begin{array}{l}\text { F5. Usar utensílios comuns para preparar refeições } \\
\text { (ex.: abridor de latas, descascador de batatas, faca } \\
\text { afiada). }\end{array}$ & 5 & 4 & 3 & 2 & 1 \\
\hline F6. Segurar um copo cheio d'água em uma mão. & 5 & 4 & 3 & 2 & 1 \\
\hline $\begin{array}{l}\text { F7. Caminhar por } 1,5 \mathrm{~km} \text {, descansando quando } \\
\text { necessário. }\end{array}$ & 5 & 4 & 3 & 2 & 1 \\
\hline $\begin{array}{l}\text { F8. Subir e descer um lance de escadas externas sem } \\
\text { usar o corrimão }\end{array}$ & 5 & 4 & 3 & 2 & 1 \\
\hline $\begin{array}{l}\text { F9. Correr distâncias curtas, como para pegar um } \\
\text { ônibus. }\end{array}$ & 5 & 4 & 3 & 2 & 1 \\
\hline $\begin{array}{l}\text { F10. Alcançar algo posicionado acima da cabeça, } \\
\text { quando de pé. }\end{array}$ & 5 & 4 & 3 & 2 & 1 \\
\hline F11. Sentar-se e levantar-se de um sofá baixo e macio. & 5 & 4 & 3 & 2 & 1 \\
\hline F12. Colocar e tirar um casaco ou uma jaqueta. & $5 \mathrm{v}$ & 4 & 3 & 2 & 1 \\
\hline $\begin{array}{l}\text { F13. Alcançar as costas, como se passasse o cinto pela } \\
\text { parte de trás das calças. }\end{array}$ & 5 & 4 & 3 & 2 & 1 \\
\hline F14. Subir e descer de um meio-fio. & 5 & 4 & 3 & 2 & 1 \\
\hline F15. Abrir uma porta externa pesada. & 5 & 4 & 3 & 2 & 1 \\
\hline $\begin{array}{l}\text { F16. Abrir um pacote de lanche (por exemplo, } \\
\text { embalagens de biscoitos) usando somente as mãos. }\end{array}$ & 5 & 4 & 3 & 2 & 1 \\
\hline F17. Servir-se de uma jarra grande. & 5 & 4 & 3 & 2 & 1 \\
\hline
\end{tabular}




\section{Questões sobre Função - Continuação...}

\begin{tabular}{|c|c|c|c|c|c|}
\hline $\begin{array}{l}\text { Quanta dificuldade você tem para...? } \\
\text { (Lembre-se que isso é sem a ajuda de outras } \\
\text { pessoas e sem o uso de qualquer dispositivo de } \\
\text { auxílio para a locomoção.) }\end{array}$ & 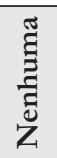 & 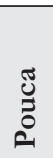 & 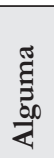 & 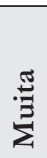 & 离 \\
\hline F18. Entrar e sair de um carro/táxi (sedan). & 5 & & & & \\
\hline $\begin{array}{l}\text { F19. Caminhar alguns quilômetros em superfícies } \\
\text { irregulares, incluindo morros. }\end{array}$ & 5 & 4 & 3 & 2 & 1 \\
\hline $\begin{array}{l}\text { F20. Subir e descer três lances de escadas internas } \\
\text { usando o corrimão. }\end{array}$ & 5 & 4 & 3 & 2 & 1 \\
\hline $\begin{array}{l}\text { F21. Levantar e mover uma cadeira da cozinha para } \\
\text { fazer a limpeza. }\end{array}$ & 5 & 4 & 3 & 2 & 1 \\
\hline $\begin{array}{l}\text { F22. Usar um banquinho para alcançar uma prateleira } \\
\text { alta. }\end{array}$ & 5 & 4 & 3 & 2 & 1 \\
\hline $\begin{array}{l}\text { F23. Arrumar a cama, incluindo esticar e prender os } \\
\text { lençóis no colchão. }\end{array}$ & 5 & 4 & 3 & 2 & 1 \\
\hline $\begin{array}{l}\text { F24. Carregar algo em ambos os braços enquanto } \\
\text { sobe um lance de escadas (por exemplo, um cesto de } \\
\text { roupas). }\end{array}$ & 5 & 4 & 3 & 2 & 1 \\
\hline $\begin{array}{l}\text { F25. Inclinar-se, a partir da posição de pé, para pegar } \\
\text { uma peça de roupa no chão. }\end{array}$ & 5 & 4 & 3 & 2 & 1 \\
\hline $\begin{array}{l}\text { F26. Circular em um andar de sua casa, considerando } \\
\text { desníveis, portas, móveis e diversos tipos de piso. }\end{array}$ & 5 & 4 & 3 & 2 & 1 \\
\hline $\begin{array}{l}\text { F27. Levantar-se do chão, a partir da posição deitada } \\
\text { (como se você estivesse deitado no chão). }\end{array}$ & 5 & 4 & 3 & 2 & 1 \\
\hline $\begin{array}{l}\text { F28. Lavar louças, panelas e utensílios em pé diante } \\
\text { da pia. }\end{array}$ & 5 & 4 & 3 & 2 & 1 \\
\hline F29. Caminhar por vários quarteirões. & 5 & 4 & 3 & 2 & 1 \\
\hline $\begin{array}{l}\text { F30. Caminhar rapidamente por } 1,5 \mathrm{~km} \text { sem parada } \\
\text { para descanso. }\end{array}$ & 5 & 4 & 3 & 2 & 1 \\
\hline F31. Subir e descer do ônibus. & 5 & 4 & 3 & 2 & 1 \\
\hline F32. Andar em uma superfície externa escorregadia. & 5 & 4 & 3 & 2 & 1 \\
\hline
\end{tabular}

\section{Questões sobre Função}

Para aqueles que usam dispositivos para a locomoção

As perguntas abaixo são apenas para pessoas que usam bengalas, andadores ou outros dispositivos de auxílio para a locomoção.

Quando você usa sua bengala, andador ou qualquer outro dispositivo para a locomoção, quanta dificuldade você tem para...?

FD7. Caminhar 1,5 km, descansando quando necessário.

FD8. Subir e descer um lance de escadas externas sem usar o corrimão.

F D14. Subir e descer de um meio-fio.

FD15. Abrir uma porta externa pesada.

FD26. Circular em um andar de sua casa, considerando desníveis, portas, móveis e diversos tipos de piso.

FD29. Caminhar por vários quarteirões.

FD30. Caminhar rapidamente por $1,5 \mathrm{~km}$ sem parada para descanso.

FD32. Andar em uma superfície externa escorregadia.

\begin{tabular}{|c|c|c|c|c|}
\hline 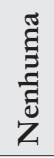 & $\begin{array}{l}\tilde{U} \\
0 \\
0 \\
0\end{array}$ & 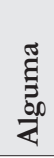 & $\stackrel{\stackrel{5}{\Xi}}{\Sigma}$ & 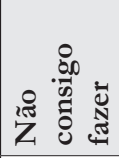 \\
\hline 5 & 4 & 3 & 2 & 1 \\
\hline 5 & 4 & 3 & 2 & 1 \\
\hline 5 & 4 & 3 & 2 & 1 \\
\hline 5 & 4 & 3 & 2 & 1 \\
\hline 5 & 4 & 3 & 2 & 1 \\
\hline 5 & 4 & 3 & 2 & 1 \\
\hline 5 & 4 & 3 & 2 & 1 \\
\hline 5 & 4 & 3 & 2 & 1 \\
\hline
\end{tabular}


Anexo 2

\section{AUXÍLIO GRÁFICO PARA RESPOSTAS DE INCAPACIDADE ${ }^{1} \# 1$}

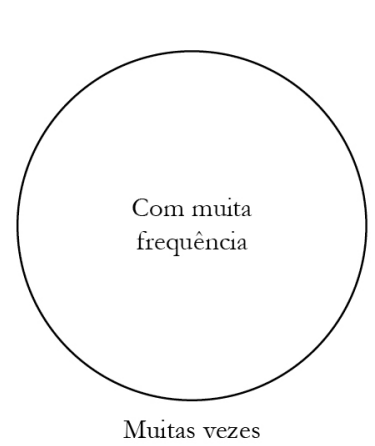

Grande parte do tempo

Ocupa uma parte

importante da sua vida

Com que frequência você...?

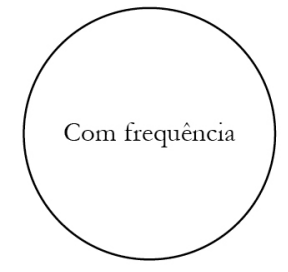

Regularmente

Uma parte regular da sua vida

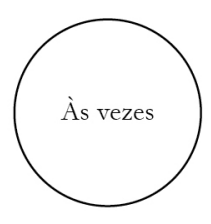

Com baixa frequência

De tempos em tempos

Ocasionalmente
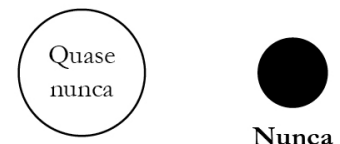

Muito poucas vezes

Raramente

\section{AUXÍLIO GRÁFICO PARA RESPOSTAS DE INCAPACIDADE \#2}

Até que ponto você se sente limitado em...?

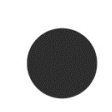

De jeito nenhum

Sem limitações

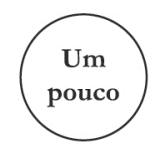

Pequenas limitações

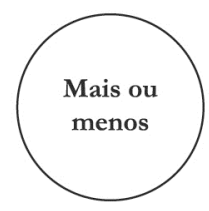

Limitações moderadas

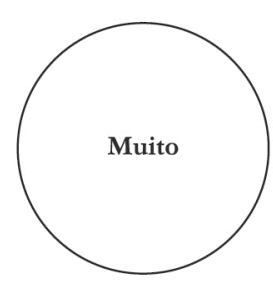

Grandes limitações

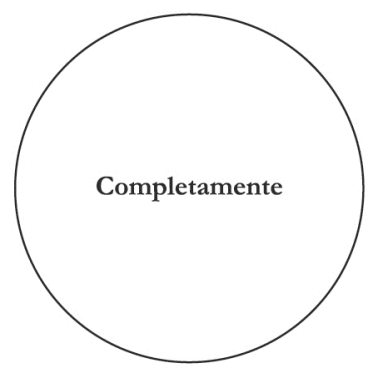

Limitação total

Não consegue fazer

Exemplos de fatores limitantes que podem limitá-lo:

- Energia mental ou física;

- Muito esforço;

- Circunstâncias sociais e econômicas;

- Problemas com transporte;

- Questões de acessibilidade;

- Saúde. 


\section{AUXÍLIO GRÁFICO PARA RESPOSTAS DE FUNÇÃO \#1}

Atualmente, quanta dificuldade você tem ao fazer a atividade sem a ajuda de outra pessoa e sem o uso de uma bengala, andador ou qualquer outro dispositivo de assistência para a locomoção?

Atualmente, quanta dificuldade você tem ao fazer a atividade sem a ajuda de outra pessoa e sem o uso de uma bengala, andador ou qualquer outro dispositivo de assistência para a locomoção?
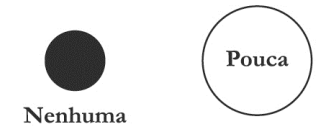

Você não tem dificuldade em fazer a atividade sozinho (a).

$$
\begin{gathered}
\text { Você pode } \\
\text { fazê-la } \\
\text { sozinho (a) } \\
\text { com um pouco } \\
\text { de dificuldade. }
\end{gathered}
$$

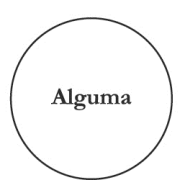

Você pode fazê-la, mas tem uma dificuldade moderada para fazê-la sozinho (a).

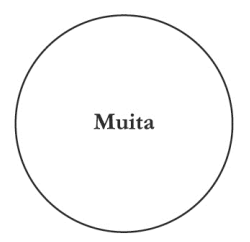

Você consegue realizá-la sem ajuda, mas tem bastante dificuldade.

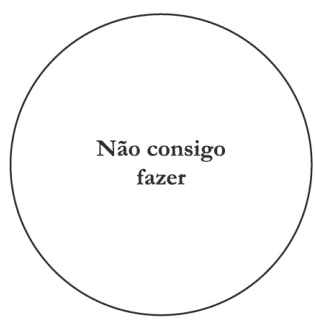

É tão difícil que você não consegue fazê-la a não ser que tenha ajuda.

Fatores que podem influenciar seu nível de dificuldade:

- Dor;

- Fadiga;

- Medo;

- Dolorimentos;

- Doenças;

- Incapacidade.

\section{AUXÍLIO GRÁFICO PARA RESPOSTAS DE FUNÇÃO \#2 (Para usuários de bengala ou outros dispositivos de assistência para a locomoção.)}

Atualmente, quanta dificuldade você tem ao fazer a atividade quando usa sua bengala, andador ou qualquer outro dispositivo de assistência para a locomoçáo?

Atualmente, quanta dificuldade você tem ao fazer a atividade quando usa sua bengala, andador ou qualquer outro dispositivo de assistência para a locomoção?
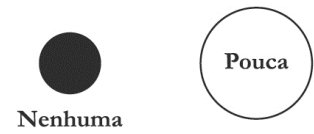

Nenhuma

Você não tem dificuldade em fazer a atividade sozinho (a).

Você pode fazê-la

sozinho (a)

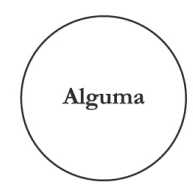

com um pouco de dificuldade.

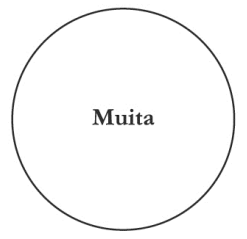

Você consegue realizá-la sem ajuda, mas tem bastante dificuldade.

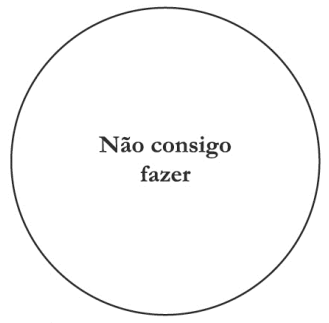

É tão difícil que você não consegue fazê-la a não ser que tenha ajuda.

Fatores que podem influenciar seu nível de dificuldade:

- Dor;

- Fadiga;

- Medo;

- Ferimentos;

- Doenças e incapacidade. 